\title{
USO DE APLICATIVOS MÓVEIS NO PROCESSO DE ENSINO-APRENDIZAGEM NA GRADUAÇÃO EM ENFERMAGEM
}

\author{
USE OF MOBILE APPLICATIONS IN THE \\ TEACHING-LEARNING PROCESS IN \\ NURSING GRADUATION
}

\section{USO DE APLICACIONES MÓVILES EN EL PROCESO DE ENSEÑANZA-APRENDIZAJE EN LA GRADUACIÓN EN ENFERMERÍA}

\author{
Tiago Ribeiro dos Santos ${ }^{1}$ \\ Luciano Gualberto Soares ${ }^{2}$ \\ Lucas Dias Soares Machado ${ }^{3}$ \\ Nayara Santana Brito ${ }^{4}$ \\ Maria Augusta Vasconcelos Palácio 5 \\ Maria Rocineide Ferreira da Silva ${ }^{6}$
}

Como citar este artigo: Santos TR, Soares LG, Machado LDS, Brito NS, Palácio MAV, Silva MRF. Uso de aplicativos móveis no processo de ensino-aprendizagem na graduação em enfermagem. Rev baiana enferm. 2021;35:e37136.

Objetivo: identificar os aplicativos móveis disponíveis para o ensino de exame-físico e procedimentos de enfermagem e como estes têm contribuído com o processo de ensino-aprendizagem na graduação de enfermagem. Método: revisão integrativa da literatura, realizada em abril de 2020, conforme protocolo pré-definido e recomendações PRISMA. Resultados: os aplicativos Oiva®, Vital Easy ${ }^{\circledR}$, Whatsapp ${ }^{\circledR}$, Facebook® e e-mails foram identificados como utilizáveis no processo de ensino-aprendizagem na graduação em enfermagem, contribuindo com maior interação entre estudantes e professores, esclarecimento de dúvidas e como fonte de informação para consultas. Conclusão: a utilização de aplicativos móveis é pertinente no processo de ensino-aprendizagem na graduação de enfermagem, por oportunizar a troca de experiências e informações entre indivíduos pertencentes a diferentes realidades, ampliando o acesso ao conteúdo, possibilitando o engajamento, limitando barreiras geográficas e adequando-se a realidades específicas.

Descritores: Aplicativos Móveis. Exame Físico. Educação em Enfermagem.

Objective: to identify the mobile applications available for the teaching of physical examination and nursing procedures and how they have contributed to the teaching-learning process in nursing graduation. Method: integrative literature review, conducted in April 2020, according to predefined protocol and PRISMA recommendations. Results:

\footnotetext{
Estudante de Enfermagem. Universidade Regional do Cariri. Iguatu, Ceará, Brasil. https://orcid.org/0000-0002-86|4-08/8.

Estudante de Enfermagem. Universidade Regional do Cariri. Iguatu, Ceará, Brasil. https://orcid.org/0000-0003-0349-3288.

Enfermeiro. Mestre em Enfermagem. Universidade Estadual do Ceará. Docente da Universidade Regional do Cariri. Crato, Ceará, Brasil. lucasdsmachado@hotmail.com. https://orcid.org/0000-0003-4450-3796.

Enfermeira. Universidade Estadual do Ceará. Docente da Universidade Regional do Cariri. Crato, Ceará, Brasil. https://orcid.org/0000-0002-9782-55।3.

Enfermeira. Doutora em Educação em Ciências e Saúde. Docente da Universidade Federal do Vale do São Francisco. Petrolina, Pernambuco, Brasil. https://orcid. org/0000-0002-2780- I 25X

Enfermeira. Doutora em Saúde Coletiva. Docente da Universidade Estadual do Ceará. Fortaleza, Ceará, Brasil. https://orcid.org/0000-0002-6086-690। .
} 
the applications Oiva ${ }^{\circledR}$, Vital Easy ${ }^{\circledR}$, Whatsapp ${ }^{\circledR}$, Facebook ${ }^{\circledR}$ and e-mails were identified as usable in the teachinglearning process in nursing graduation, contributing to greater interaction between students and professors, clarification of doubts and as a source of information for lookup. Conclusion: the use of mobile applications is relevant in the teaching-learning process in nursing graduation, because they provide opportunities for the exchange of experiences and information between individuals belonging to different realities, expanding access to content, enabling engagement, limiting geographical barriers and adapting to specific realities.

Descriptors: Mobile Applications. Physical Examination. Education, Nursing.

Objetivo: identificar las aplicaciones móviles disponibles para la enseñanza de los procedimientos de examen físico y procedimientos de enfermería y cómo han contribuido al proceso de enseñanza-aprendizaje en la graduación en enfermería. Método: revisión integrativa de la literatura, realizada en abril de 2020, de acuerdo con el protocolo predefinido y las recomendaciones PRISMA. Resultados: las aplicaciones Oiva ${ }^{\circledR}$, Vital Easy ${ }^{\circledR}$, Whatsapp ${ }^{\circledR}$, Facebook ${ }^{\circledR}$ y correos electrónicos fueron identificados como utilizables en el proceso de enseñanza-aprendizaje en la graduación enfermería, contribuyendo con una mayor interacción entre estudiantes y profesores, aclarando dudas y como fuente de información para consultas. Conclusión: el uso de aplicaciones móviles es relevante en el proceso de enseñanza-aprendizaje en la graduación en enfermería, ya que proporciona oportunidades para el intercambio de experiencias e información entre personas pertenecientes a diferentes realidades, ampliando el acceso a los contenidos, permitiendo el compromiso, limitando las barreras geográficas y adaptándose a realidades especificas.

Descriptores: Aplicaciones Móviles. Examen Físico. Educación en Enfermería.

\section{Introdução}

Os processos de viver e produzir na atualidade, influenciados pela globalização e aproximação entre indivíduos e nações mediados pelo advento da internet, têm demandado constante atualização e consumo de informações. O acesso à informação está oportunizado pelo uso comum e rotineiro de celulares, computadores e outros instrumentos com sistemas de informação conectados à internet, fomentando a união da vida na sociedade atual e o uso de tecnologias digitais de informação e comunicação (TDIC). No âmbito da educação, a utilização dessas ferramentas no processo de ensino-aprendizagem fortalece a construção de conhecimentos, ampliando o potencial desse processo ${ }^{(1)}$. Ademais, promove autoria, participação ativa e colaboração do educando, uma vez que a possibilidade de aprender nesses ambientes mediados pelas TDIC é capaz de reconfigurar os caminhos percorridos pelos estudantes para adquirir conhecimento ${ }^{(2)}$.

No campo da formação em saúde, observam-se inúmeras inovações nos métodos de ensino e aprendizagem para melhor atender às demandas de aprendizagem dos educandos, dentre elas a incorporação das TDIC dentro e fora da sala de aula, com diversificação de temáticas e aplicabilidades em diferentes contextos de formação( ${ }^{(3)}$.

As TDIC compreendem os avanços trazidos pelos recursos e ferramentas digitais que agregaram novas formas de interação e comunicação às tecnologias de informação e comunicação (TIC), que incluem os dispositivos eletrônicos e tecnológicos, como computador e internet ${ }^{(4)}$. As TDIC, por sua vez, representam o universo promovido pela Web 2.0 e suas ferramentas digitais, permitindo ao usuário maior interação e produção de conteúdo, tais como blogs, redes sociais e softwares educacionais ${ }^{(5)}$.

Dentre as TDIC, destaca-se o desenvolvimento e uso de aplicativos móveis (apps), configurando um agrupamento de recursos tecnológicos produzidos com base em um parâmetro científico para assegurar maior atratividade e comunicação aos procedimentos formativos ${ }^{(6)}$. O uso desses aplicativos adquiriu espaço na vida das pessoas, influenciando de modo direto nas políticas públicas e movendo a sociedade na direção de modernos conhecimentos acerca dos valores democráticos e socioculturais, empreendendo função de recurso educacional e informativo. Dessa forma, a construção das relações 
dos indivíduos com seu processo de formação profissional é fortemente influenciada pelo uso dessas tecnologias, sendo relevante estimular a sua integração pedagógica nos variados panoramas da formação em saúde ${ }^{(7)}$.

No que se refere à área da enfermagem, convém contextualizar que o desenvolvimento e a aplicabilidade desses recursos tecnológicos concorrem positivamente na construção e disseminação de conhecimentos, uma vez que a aprendizagem das técnicas básicas comumente é pontuada pelos acadêmicos como um período misto de identificação e realização profissional, mas também com angústia e medo em virtude do desconhecimento e da inexperiência. Assim, esse tipo de tecnologia fomenta um processo educacional mais atrativo e interativo ${ }^{(8)}$.

O cenário mediado pelo uso desses aplicativos oferece novas possibilidades pedagógicas inerentes ao ambiente virtual, promovendo participação ativa dos discentes e maior interação entre estes e os docentes, contribuindo com práticas inovadoras e alinhadas às demandas atuais para o ensino da enfermagem. Nessa perspectiva, os recursos tecnológicos fomentam a descentralização do trabalho pedagógico, possibilitando novas formas de construir o conhecimento por meio da interatividade entre o aluno e o professor $^{(6)}$.

A integração dessas tecnologias ao processo de formação de profissionais enfermeiros torna-se um objeto relevante, por demonstrar potencial para o favorecimento do engagement, da participação, da curiosidade, da interação aluno-professor e aluno-aluno. Nesse sentido, objetivou-se identificar os aplicativos móveis disponíveis para o ensino de exame-físico e procedimentos de enfermagem e como estes têm contribuído com o processo de ensino-aprendizagem na graduação de enfermagem.

\section{Método}

Trata-se de uma revisão integrativa da literatura, que buscou identificar, analisar e sintetizar evidências sobre o uso de aplicativos móveis para o processo de ensino-aprendizagem na graduação em enfermagem. Para sua operacionalização, construiu-se um protocolo de pesquisa contemplando as etapas de elaboração da pergunta de revisão, busca e seleção dos estudos primários, extração de dados dos estudos, avaliação crítica dos estudos primários incluídos, síntese dos resultados e apresentação da revisão ${ }^{(9)}$.

$\mathrm{Na}$ primeira etapa, para operacionalização da busca das referências que compuseram a revisão em questão, utilizou-se a estratégia PICO para formulação da questão norteadora da pesquisa: Quais os aplicativos móveis disponíveis para ensino de exame-físico e procedimentos de enfermagem e como estes têm contribuído com o processo de ensino-aprendizagem na graduação de enfermagem.

Essa definição permitiu iniciar-se, em abril de 2020, a busca do material para análise, de modo pareado, por meio do portal da Coordenação de Aperfeiçoamento de Pessoal de Nível Superior (CAPES) na base de dados Latin American and Caribbean Health Sciences Literature (LILACS), do portal Public Medical Literature Analysis and Retrieval System Online (PubMed) e da biblioteca eletrônica Scientific Electronic Library Online (SciELO).

Os cruzamentos de busca foram articulados por meio da relação dos descritores selecionados nos Descritores em Ciências da Saúde (DeCS) e no Medical Subjetc Headings (MeSH), a saber: aplicativos móveis (mobile applications), exame físico (physical examination) e educação em enfermagem (education, nursing). Esses descritores foram associados pelos operadores booleanos AND e OR e acrescidos de termos sinônimos para ampliar a sensibilidade das buscas, estruturando-se quatro equações:

a) ("Aplicativos móveis" OR "Apps móveis") AND ("educação em enfermagem" OR "ensino de enfermagem" OR "estudantes de enfermagem");

b) ("Mobile applications" OR "Apps, Mobile" OR "Mobile App") AND ("Education, Nursing" OR "Nursing Education" OR "Educations, Nursing"); 
c)"Exame físico" AND ("Aplicativos móveis" OR "Apps móveis"); e

d)("Physical Examination" OR "Examinations, Physical" OR "Physical Examinations") AND ("Mobile applications" OR “Apps, Mobile" OR "Mobile App").

Operacionalizando as buscas, delimitou-se como critérios de inclusão: estudos publicados no período de 2015 a 2020, em português, inglês e/ou espanhol, disponíveis na íntegra e que contemplassem o uso de aplicativos no processo de formação de enfermeiros, objeto do estudo.
A delimitação temporal para busca dos estudos considerou o recente avanço na implementação das estratégias da Política Nacional de Ciência, Tecnologia e Inovação em Saúde e o advento do uso de TDIC no ensino na área de saúde.

Foram excluídos artigos que não tratavam da graduação em enfermagem e/ou uso de aplicativos nessa formação. O processo de identificação, triagem, elegibilidade e inclusão está sumarizado, conforme o instrumento Preferred Reporting Items for Systematic Review and Meta-Analyses (PRISMA), ilustrado na Figura 1.

Figura 1 - Processo de seleção dos estudos para análise

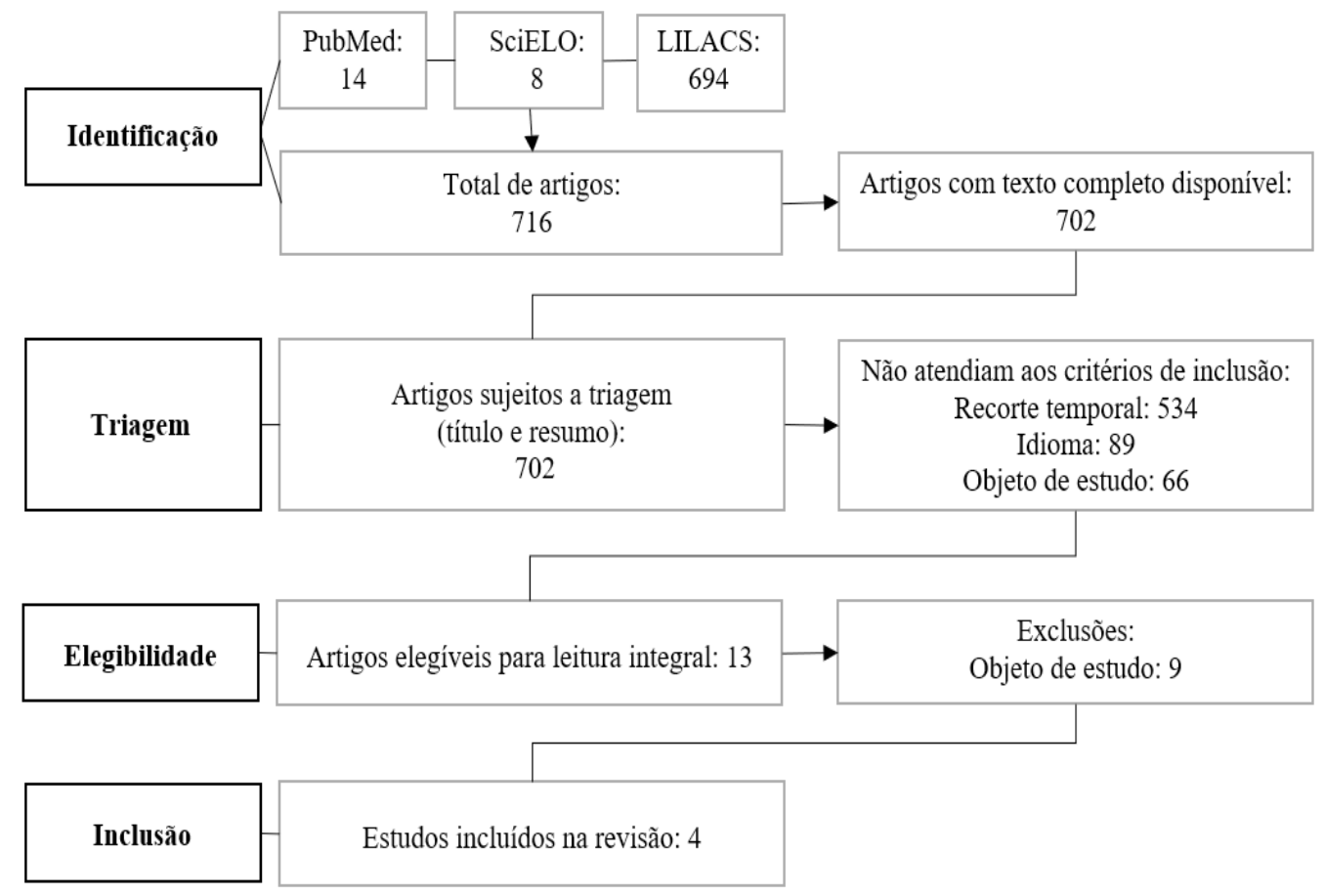

Fonte: Elaboração própria.

Após a seleção dos estudos que comporiam a revisão, na terceira etapa, foram extraídos os dados dos estudos, sumarizando-os com base em um instrumento elaborado pelos pesquisadores, contendo as variáveis de interesse: tema abordado, autoria, país, aplicativo, características do aplicativo pertinentes à formação em enfermagem e tipo de estudo.

Para a avaliação metodológica dos artigos selecionados, optou-se pelo uso do Critical Appraisal Skills Programe (CASP), que permite qualificar sistematicamente a confiabilidade, a relevância e os resultados dos trabalhos publicados. Nesse sentido, os estudos foram classificados quanto à adequação metodológica e riscos de viés em A, para aqueles com qualidade metodológica boa, e viés reduzido, quando, na avaliação, o escore encontrava-se entre seis e dez pontos; e viés em B, quando foi satisfatória a qualidade metodológica, mesmo com um risco de viés aumentado, representando aqueles com escore abaixo de seis pontos. Esse processo 
envolve questionamentos, tais como: Qual a questão do estudo? A questão do estudo foi respondida? O estudo atingiu seus objetivos?

As etapas de busca e seleção da literatura, extração dos dados e avaliação crítica dos estudos foi realizada concomitantemente por dois pesquisadores. Em caso de divergências, um terceiro pesquisador realizou a avaliação, contribuindo para o desembaraço da decisão. Por fim, os dados foram sintetizados, apresentados e discutidos com a literatura pertinente. Para assegurar os direitos éticos e legais relacionados à autoria e condução das publicações analisadas, fez-se referência aos devidos autores, ao mencionar seus achados.

\section{Resultados}

Os estudos que compuseram a amostra analisada foram publicados no período de 2017 a 2020 e nos idiomas inglês e português. No que tange ao nível de evidência, todos foram classificados como pertencentes à classe A, demonstrando estruturação metodológica adequada em sua condução. O Quadro 1 apresenta um panorama geral das publicações analisadas, contemplando o tema trabalhado, a autoria, o país de origem, o aplicativo móvel utilizado e o tipo de estudo.

Quadro 1 - Informações sobre os artigos incluídos na revisão

\begin{tabular}{|l|l|l|l|l|}
\hline Tema trabalhado & \multicolumn{1}{|c|}{ Autoria } & \multicolumn{1}{|c|}{ País } & \multicolumn{1}{|c|}{ Aplicativo } & Tipo de estudo \\
\hline $\begin{array}{l}\text { Aplicabilidade de dispositivo } \\
\text { móvel no tratamento de } \\
\text { transtorno mental }\end{array}$ & $\begin{array}{l}\text { Kaipainen K, } \\
\text { Välkkynen P, } \\
\text { Kilkku N }\end{array}$ & Finlândia & OIVA & $\begin{array}{l}\text { Estudo } \\
\text { reflexivo }\end{array}$ \\
\hline $\begin{array}{l}\text { Teoria e prática clínica do } \\
\text { curso de enfermagem através } \\
\text { da exploração de dispositivos } \\
\text { móveis }\end{array}$ & $\begin{array}{l}\text { Willemse JJ, } \\
\text { Bozalek V }\end{array}$ & $\begin{array}{l}\text { África do } \\
\text { Sul }\end{array}$ & $\begin{array}{l}\text { E-mail, SMS, } \\
\text { WhatsApp e } \\
\text { Facebook }\end{array}$ & $\begin{array}{l}\text { Estudo } \\
\text { exploratório, } \\
\text { descritivo }\end{array}$ \\
\hline $\begin{array}{l}\text { O uso do WhatsApp para } \\
\text { melhorar a educação primária } \\
\text { na saúde }\end{array}$ & Willemse JJ & $\begin{array}{l}\text { África do } \\
\text { Sul }\end{array}$ & WhastApp & $\begin{array}{l}\text { Estudo } \\
\text { exploratório, } \\
\text { descritivo }\end{array}$ \\
\hline $\begin{array}{l}\text { Construção de um aplicativo } \\
\text { digital para o ensino de } \\
\text { sinais vitais }\end{array}$ & $\begin{array}{l}\text { Pereira FGF, } \\
\text { Rocha DJL, } \\
\text { Melo GAA, } \\
\text { Jaques RMPL, } \\
\text { Formiga LMF }\end{array}$ & Brasil & Vital Easy & $\begin{array}{l}\text { Estudo } \\
\text { metodológico }\end{array}$ \\
\hline
\end{tabular}

Fonte: Elaboração própria.

Dos aplicativos móveis identificados, apenas o Vital Easy ${ }^{(6)}$ foi construído especificamente para o ensino de atributos referentes à prática de enfermagem, voltando-se essencialmente para as técnicas de aferição e interpretação de sinais vitais. Esta ferramenta tecnológica foi construída conforme referencial de Galvis-Panqueva, em suas etapas de análise, desenho e desenvolvimento, não sendo apontada sua validação por especialistas ou público-alvo.

O aplicativo OIVA ${ }^{(10)}{ }^{(1)}$ embora possa ser utilizado no aprendizado de questões de saúde mental na graduação em enfermagem, não se destina essencialmente a este propósito, sendo adotado para desenvolvimento de habilidades para lidar com a depressão. Não há, no estudo analisado, a descrição do processo de construção dessa tecnologia.

As mídias sociais e de contato habitual apresentaram potencialidades quanto ao uso em processos formativos, em decorrência de fatores, como fácil acesso, gratuidade e interação possibilitada entre os atores do ensino-aprendizagem, como docentes e discentes ${ }^{(11-12)}$. Percebeu-se 
maior identificação com o e-mail, dentre as possibilidades em dispositivos móveis, visto que este tipo de aplicativo móvel já compõe o acervo dos smartphones atuais e possibilita compartilhar arquivos em maior tamanho e de formatos diversos, como textos, imagens, vídeos, softwares, entre outros ${ }^{(11)}$.

O WhatsApp ${ }^{\circledR}$ foi apontado como recurso para realização de grupos de discussão, oportunizando o acesso a informações em tempo e local oportuno, conforme necessidade, assegurando orientação para a prática, fonte de informações para os testes e esclarecimentos de dúvidas ${ }^{(12)}$. O Facebook ${ }^{\circledR}$ foi reconhecido enquanto possibilidade de promover o envolvimento entre estudantes, bem como com a comunidade, tendo em vista o frequente uso desta tecnologia ${ }^{(11)}$.

A Figura 2 condensa os principais aspectos referentes ao uso no processo de ensino-aprendizagem dos aplicativos apontados nos estudos.

Figura 2 - Aspectos referentes ao ensino-aprendizagem no uso dos aplicativos digitais identificados na literatura

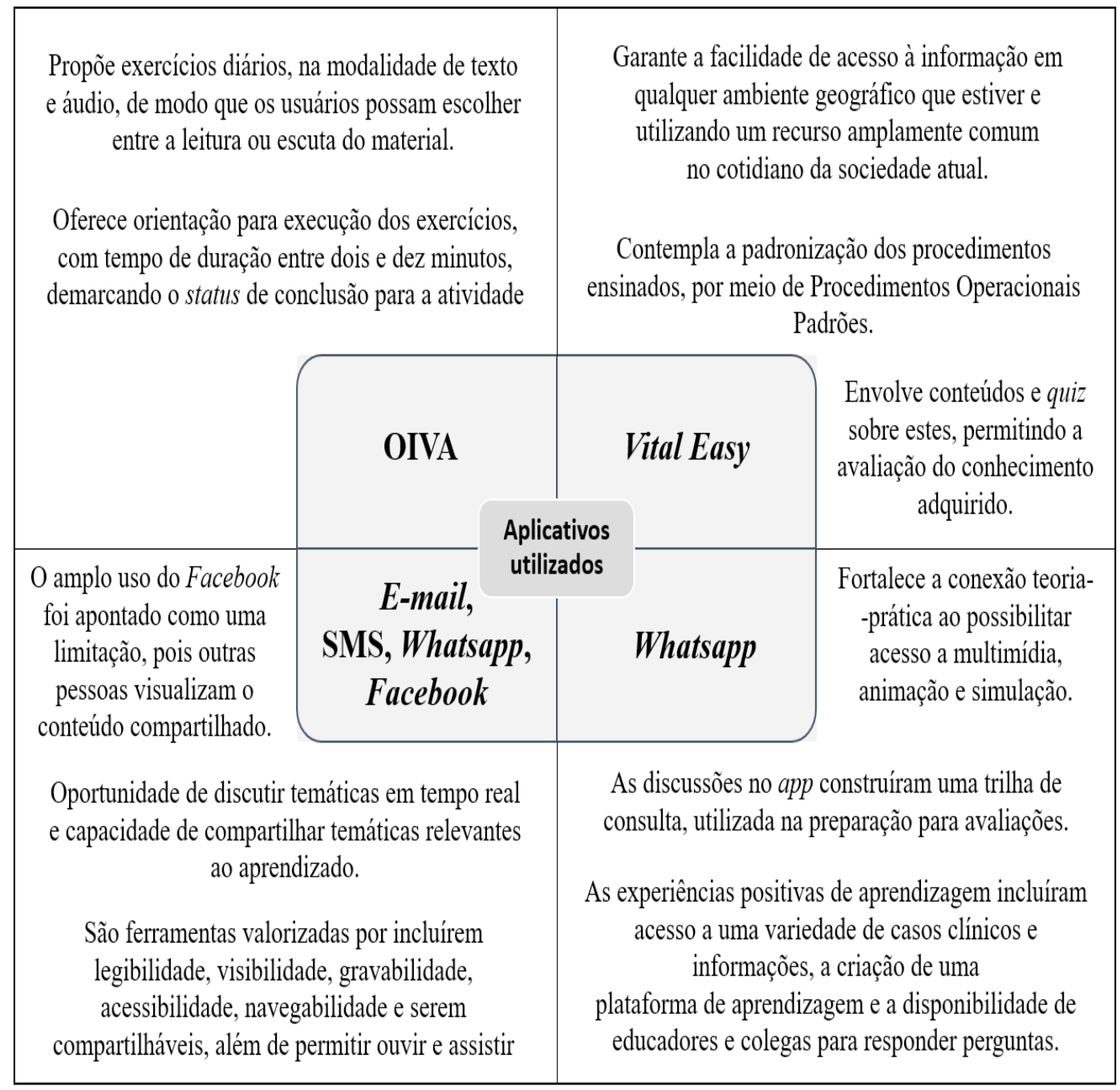

Fonte: Elaboração própria.

Alguns desafios são apontados para o uso de aplicativos móveis no processo de ensino-aprendizagem, dentre eles a necessidade de validação por juízes e público-alvo e a adoção de vias institucionais, de modo a assegurar questões formais do ensino, como não compartilhamento de material, opiniões e posicionamentos com pessoas externas à realidade de ensino ${ }^{(11)}$. 


\section{Discussão}

As adequações do processo de ensino-aprendizagem, a fim de prover uma resposta mais adequada às demandas sociais e necessidades formativas, têm solicitado atualizações nas estratégias pedagógicas, ferramentas didáticas e habilidades educacionais, acrescendo a dinâmica e utilização da tecnologia para proporcionar uma aprendizagem significativa ${ }^{(14)}$. Nesse contexto, aplicativos móveis demonstram seu potencial em flexibilizar o ensino e promover aprendizagens ativas, respondendo à necessidade dos usuários e materializando-se como inovação na formação profissional.

O uso de aplicativos móveis na formação em enfermagem é concernente à contemporaneidade da profissão e sua afirmação enquanto ciência, adotando tecnologias educacionais capazes de promover o conhecimento teórico e o pensamento crítico e reflexivo necessários ao maior diálogo entre teoria e prática e ao desenvolvimento de competências ${ }^{(13)}$.

A utilização de TDIC, no que tange aos aspectos de ensino e aprendizagem por meio de aplicativos móveis, tem como finalidade melhorar o aprendizado dos alunos. Tem se tornando usual no ensino superior, sobretudo, contribuindo efetivamente com as aulas práticas. As TDIC permeiam o cotidiano de discentes e docentes e oferecem acesso abundante à comunicação e informação. Educadores e alunos podem utilizar essas ferramentas em diversos contextos com propósitos de aprendizado, por exemplo, fóruns de discussão e distribuição de conteúdo ${ }^{(12)}$.

Os aplicativos identificados nos estudos fomentam contribuição ao processo de ensino-aprendizagem no cenário de formação superior de enfermeiros, enfatizando que a produção e/ou avaliação desses recursos tecnológicos, no âmbito do ensinar e aprender, proporciona maior relação entre educandos e educadores dentro do cenário digital.

Existem inúmeras possibilidades de integração de tecnologias ao ensino, inclusive no ensino de enfermagem ${ }^{(15)}$. Dentre essas, destaca-se o uso crescente de aplicativos móveis, justificado pela sua praticidade de acesso e disponibilidade na atual conjuntura tecnológica em que o mundo está inserido, podendo ser acessado em ambiente extraclasse, como um complemento do ensino. Além disso, o ambiente universitário torna-se propício para aplicação de apps móveis, uma vez que podem ser criados e executados pelos próprios acadêmicos, possibilitando aquisição de conhecimentos e de habilidades também nesse processo ${ }^{(16)}$.

Os apps desenvolvidos no espaço universitário, pelos próprios docentes, alinhados ao componente curricular e apoiado na intercolaboração com discentes, adéquam-se melhor às práticas educativas por estruturarem um ambiente mais favorável e atrativo, potencializando e facilitando o engajamento nessas ações ${ }^{(17)}$.

Ao incorporar aplicativos móveis no processo de ensino-aprendizagem na área da saúde, os educadores estão inovando na relação entre ensinar e aprender, à medida que se adaptam à necessidade de modelos educacionais contemporâneos. Nessa perspectiva, a enfermagem segue essa renovação, por meio do uso de ambientes virtuais, viabilizando a interatividade e favorecendo o processo de aprendizagem ${ }^{(18)}$.

A inclusão de ambientes digitais em cursos de graduação reserva ao aluno autonomia na aprendizagem, além de fomentar a inclusão digital dos envolvidos, tornando o processo mais dinâmico e motivador ${ }^{(16)}$. Demonstra ainda possibilidade de uso em situações que inviabilizam o contato direto com o docente, como realização de atividades no ambiente domiciliar, desenvolvimento de ações na comunidade e períodos de aulas remotas.

É relevante discutir que a ampliação do uso de TDIC, tais como os apps, não representa um processo de robotização da educação e substituição do ser docente no processo de ensino-aprendizagem, visto que essas ferramentas didáticas exigem um preparo organizacional, adequação metodológica e referencial teórico apropriado. Seu bom uso depende ainda da interação adequada entre docentes e discentes, alicerçando-se como recursos complementares 
e não substitutivos aos momentos presenciais da formação ${ }^{(19)}$. Não é a simples integração das TDIC que poderá transformar as práticas pedagógicas, mas determinados usos dessas tecnologias, principalmente a sua integração contextualizada a cada realidade ${ }^{(20)}$.

Dentre os aspectos positivos do uso de aplicativos móveis, pontua-se o rompimento de barreiras de acesso à educação e contextos de aprendizagem inovadores, suprindo distâncias geográficas, carências didáticas de docentes e déficits de materiais educativos, fazendo com que o processo de ensino seja mais atrativo, completo e dinâmico ${ }^{(7)}$.

Destarte, aplicativos podem ser efetivos por caracterizarem-se enquanto instrumentos inovadores no ensino na área da saúde, apresentando-se como ferramentas capazes de despertar o interesse e a motivação em aprender, haja vista que os apps móveis são utilizados em uma proporção entre $45 \%$ e $85 \%$ a mais que as consultas a livros e revistas ${ }^{(21)}$.

Defende-se que a criação de objetos educacionais digitais é sempre avaliada positivamente, quando se leva em consideração que os indivíduos estão intrinsecamente ligados a um mundo globalizado, viabilizando facilidades de comunicação e de acesso a pesquisas adicionais que o ambiente virtual disponibiliza. Entretanto, há premissas para usos desses objetos nas práticas educativas, como a formulação de acordo com a necessidade, uma estruturação conforme recomendações de especialistas e levantamento da aceitação dos usuários ${ }^{(18)}$.

Com base nessas premissas, o uso de e-mails, WhatsApp ${ }^{\circledR}$ e Facebook®, embora com potencialidades evidenciadas nos estudos analisados quanto ao seu uso e possibilidades na formação em enfermagem, não atendem às recomendações defendidas anteriormente, de construção, adequação e validação. Deste modo, devem ser adotados de modo secundário a outras ferramentas e estratégias primárias.

No tocante ao uso do app Whatsapp ${ }^{\circledR}$ em processos formativos de outras profissões da saúde, como a medicina, considerou-se a capacidade desta tecnologia na transferência de informações, como textos, áudios, imagens e vídeos, possibilitando a interação síncrona e em tempo real entre os indivíduos. Além do mais, é efetivo para a consolidação de conceitos teóricos já introduzidos no eixo de discussões com os discentes, promovendo debates, ampliando possibilidades para aplicação do conteúdo e fortalecendo a visão crítica frente às realidades do território ${ }^{(19)}$.

O Facebook, por sua vez, é reconhecido como ambiente oportuno às intervenções em saúde, podendo auxiliar no envolvimento, responsabilidade e empoderamento dos usuários, condições estas essenciais para a mudança de comportamento $^{(22)}$. Entretanto, alguns estudantes de enfermagem que experimentaram esta ferramenta na formação não a consideraram a melhor opção, pelo fácil compartilhamento de posts, curtidas e comentários com indivíduos que não pertencem ao ambiente educacional em questão, inibindo a participação ou restringindo-a ${ }^{(11)}$.

Estudo de revisão sistemática da literatura realizado anteriormente aponta que os aplicativos digitais desenvolvidos na área da saúde, nela inclusa a enfermagem, abordam aspectos de diagnóstico, telemedicina, simulação cirúrgica, treinamento, coleta de dados, educação de pacientes, comportamentos e planejamento cirúrgico $^{(23)}$. Nota-se que a construção de apps voltados para a educação de estudantes de graduação em suas dimensões teóricas, teórico-práticas e práticas ainda é incipiente.

No que concerne à educação, o entusiasmo pelo progresso e aplicação de recursos digitais na graduação demonstra crescimento e expansão, tendo em vista a disseminação de softwares educacionais em diversos cursos e disciplinas, no qual o centro é a melhoria de ensino no processo de formação dos estudantes. Essa disseminação encontra-se inserida nos contextos de desenvolvimento de ações em educação e saúde, aprendizagem de conteúdos teóricos e práticos e no protagonismo do discente no processo de aprender ${ }^{(24)}$.

Por fim, suscita a consideração de que softwares e aplicativos digitais não podem ser idealizados como a melhor ou a única alternativa para o ensino, mas de modo complementar 
ao ensino presencial, posto que o uso dessas tecnologias, mesmo de modo adequado, não assegura a eficácia da aprendizagem em meio à sua complexidade, influenciada pela forma didática de apresentação das informações, bem como interesse manifestado pelos alunos em aprender ${ }^{(13)}$.

Vale reconhecer ainda as limitações de acesso a essas tecnologias, que ainda perduram em diversas regiões, como instabilidade no acesso à internet, ausência de infraestrutura de laboratórios de informática e a limitação de habilidades no manuseio de aplicativos por parte de docentes e discentes menos familiarizados com as tecnologias ${ }^{(17)}$. No tocante ao acesso à internet na América Latina e Caribe, identificam-se países, como Nicarágua e Guatemala, com baixíssimo nível de acesso por parte da população, alcançando 10\% dos indivíduos, ao passo que países como Brasil, Chile e Argentina apresentam porcentagem de acesso próxima ou ligeiramente superior a 50\%. Países europeus e Estados Unidos possuem taxa de acesso à internet igual ou superior a $70 \%{ }^{(25)}$.

Com o avanço dos métodos educacionais e da tecnologia cada vez mais presente no ensino dos estudantes de enfermagem, espera-se estimular a construção, validação e avaliação de aplicativos digitais pertinentes ao ensino de enfermagem, principalmente na graduação, podendo beneficiar os educandos com o incentivo na busca por novos conhecimentos, além de expandir o uso de tecnologias tão presentes em outras esferas da vida.

Pode-se pontuar como limitação desta revisão a pequena quantidade de aplicativos digitais analisados, convergente com a baixa produção desses para fins educativos na formação em enfermagem, e a ausência de estudos quase-experimentais que avaliem a utilização dessas tecnologias junto a educadores e educandos, a fim de aperfeiçoá-las e inspirar outros aplicativos em processo de construção.

\section{Conclusão}

O modo de ensino e aprendizagem em enfermagem é complexo e exige, de todos os envolvidos, posturas ativas na procura por inovações para que o conhecimento e as habilidades necessárias sejam sempre úteis na tomada de decisão. Na contemporaneidade, as TDIC podem contribuir para mudanças relevantes nas formas de ensinar e aprender de alunos e professores no desenvolvimento de uma aprendizagem mais significativa, colocando em prática novas formas de ensinar e aprender na formação de profissionais de enfermagem, tão necessárias às demandas sociais atuais e ao rompimento com o modelo tradicional de ensino.

Os resultados apresentados revelam essas possibilidades de integração de TDIC, principalmente em contextos de utilização voltados para práticas específicas no ensino de enfermagem. A fundamentação e disseminação da utilização de aplicativos móveis tornam-se pertinentes, por oportunizar a troca de experiências e informações entre indivíduos pertencentes a diferentes realidades, ampliar o acesso a conteúdo, o engajamento, além de limitar barreiras geográficas e adequar-se a realidades específicas.

Assim, recomenda-se que a avaliação, pelos cursos de graduação em enfermagem, dos recursos mais adequados e efetivos para serem utilizados na sua realidade, associando a melhor tecnologia ao melhor método de ensino e às características do corpo discente, bem como a aproximação entre trabalhadores e pesquisadores das áreas de informática, educação e saúde, possam promover o desenvolvimento de aplicativos móveis condizentes com a realidade.

\section{Colaborações:}

1 - concepção, projeto, análise e interpretação dos dados: Tiago Ribeiro dos Santos, Luciano Gualberto Soares e Lucas Dias Soares Machado;

2 - redação do artigo e revisão crítica relevante do conteúdo intelectual: Tiago Ribeiro dos Santos, Luciano Gualberto Soares, Lucas Dias Soares Machado, Nayara Santana Brito, Maria Augusta Vasconcelos Palácio e Maria Rocineide Ferreira da Silva;

3 - aprovação final da versão a ser publicada: Lucas Dias Soares Machado, Nayara Santana Brito, Maria Augusta Vasconcelos Palácio e Maria Rocineide Ferreira da Silva. 


\section{Referências}

1. Barra DCC, Paim SMS, Dal Sasso GTM, Colla GW. Métodos para desenvolvimento de aplicativos móveis em saúde: revisão integrativa da literatura. Texto ContextoEnferm. 2017;26(4):e2260017. DOI: http://dx.doi. org/10.1590/0104-07072017002260017

2. Lima MTGA. Do behaviorismo ao conectivismo: reflexões sobre metodologias ativas na aprendizagem no UNIPTAN. In: Neves VJ, Mercanti LB, Lima MT. Metodologias ativas: perspectivas teóricas e práticas no ensino superior. Campinas (SP): Pontes Editores; 2018. p. 15-32.

3. Silva I, Ângelo J, Santos F, Lumini MJ, Martins T. Satisfação e usabilidade de uma tecnologia de informação e comunicação no ensino de enfermagem: um estudo piloto. Rev Enferm Referência. 2019;4(21):143-50. DOI: https://doi. org/10.12707/RIV19013

4. Costa SRS, Duqueviz BC, Pedroza RLS. Tecnologias digitais como instrumentos mediadores da aprendizagem dos nativos digitais. Psicol Esc Educ. 2015;19(3):603-10. DOI: http://dx.doi. org/10.1590/2175-3539/2015/0193912

5. Cerigatto MP. Tecnologias digitais na prática pedagógica. Porto Alegre: SAGAH; 2018.

6. Pereira FGF, Frota NM, Silva DV, Sousa LMO, Almeida JC, Cysne Filho FMS. Avaliação de aplicativo digital para o ensino de sinais vitais. Rev Min Enferm. 2017;21:e-1034. DOI: http://www. dx.doi.org/10.5935/1415-2762.20170044

7. Araújo RPA, Penteado CLC, Santos MBP. Democracia digital e experiências de e-participação: webativismo e políticas públicas. Hist Ciênc Saúde, Manguinhos. 2015;22:1597-619. DOI: http://dx.doi. org/10.1590/S0104-59702015000500004

8. Silva PS, Ramos RL, Cunha KCS, Figueiredo NMA. Habilidades e destreza manual: tratando da criação no ensino de semiotécnica na enfermagem. Rev Pesqui Cuid Fundam online. 2015;7(3):2874-86. DOI: $10.9789 / 2175-5361.2015 . v 7 i 3.2874-2886$

9. Mendes KDS, Silveira RCCP, Galvão CM. Use of the bibliographic reference manager in the selection of primary studies in integrative revies. Texto Contexto-enferm. 2019;19:e20170204. DOI: https:// doi.org/10.1590/1980-265x-tce-2017-0204

10. Kaipainen K, Välkkynen P, Kilkku N. Applicability of acceptance and commitment therapy-based mobile app in depression nursing. Transl
Behav Med. 2017;7(2):242-53. DOI: 10.1007/ s13142-016-0451-3

11. Willemse JJ, Bozalek V. Exploration of the affordances of mobile devices in integrating theory and clinical practice in an undergraduate nursing programme. Curationis. 2016;38(2):1510. DOI: 10.4102/curationis.v38i2.1510

12. Willemse JJ. Undergraduate nurses reflections on Whatsapp use in improving primary health care education. Curationis. 2015;38(2):1512. DOI:10.4102/curationis.v38i2.1512

13. Pereira FGF, Rocha DJL, Melo GAA, Jaques RMPL, Formiga LMF. Construção e validação de aplicativo digital para ensino de instrumentação cirúrgica. Cogitare enferm. 2019;24:e58334. DOI: http://dx.doi.org/10.5380/ce.v24i0.58334

14. Mota NP, Vieira CMA, Nascimento MNR, Bezerra AM, Quirino GS, Félix NDC. Mobile application for the teaching of the International Classification for Nursing Practice. Rev Bras Enferm. 2019;72(4):1020-7. DOI: http://dx.doi. org/10.1590/0034-7167-2018-0751

15. Gonçalves LBB, Pinto AGA, Duavy SMP, Faustino RS, Alencar APA, Palácio MAV. O uso das Tecnologias Digitais de Informação e Comunicação como Recurso Educacional no Ensino de Enfermagem. EaD em Foco. 2020;10(1):e939. DOI: https://doi. org/10.18264/eadf.v10i1.939

16. Pereira FGF, Silva DV, Sousa LMO, Frota NM. Construção de um aplicativo digital para o ensino de sinais vitais. Rev Gaúcha Enferm. 2016 jun;37(2):e59015. DOI: http://dx.doi. org/10.1590/1983- 1447.2016.02.59015

17. MeloJúnior PMR, Maia SMAS, Cunha RSC, Espíndola Júnior E, Bollela VR. Sala de aula invertida para o ensino do conteúdo abertura coronária em Endodontia. Revi ABENO. 2018;18(2):182-91. DOI: http://dx.doi.org/10.30979/rev.abeno.v18i2.584

18. Pereira EBF, Modesto BCM, Valença MP, Silva Junior WS, Souza CFQ. Development and assessment of a virtual learning environment for training in malignant hyperthermia. Rev Sobecc. 2017;22(4):180-7. DOI: 10.5327/ Z1414-4425201700040002

19. Paulino DB, Martins CCA, Raimondi GA, Hattori WT. WhatsApp ${ }^{\circledR}$ como recurso para a educação em saúde: contextualizando teoria e prática em um novo cenário de ensino-aprendizagem. Rev Bras Educ Médica. 2018;42(1):169-78. DOI: http://dx.doi. org/10.1590/1981-52712018v42n1RB20170061 
20. Lalueza J, Camps ICS. As tecnologias da informação e da comunicação e os processos de desenvolvimento e socialização. In: Coll C, Monereo C, organizadores. Psicologia da Educação Virtual: aprender e ensinar com as tecnologias da informação e da comunicação. Porto Alegre: Artmed; 2010. p. 47-65.

21. Chaves MJC, Barbosa ES, Nóbrega-Therrien SM. Facebook como ambiente virtual de aprendizagem no curso de enfermagem. Educa Rev Multidisc Educ. 2020;7(17):143-64. DOI: $10.26568 / 2359-2087.2020 .4275$

22. Ribeiro RM, Bragiola JVB, Eid LP, Ribeiro RCHM, Sequeira CAC, Pompeo DA. Impact of an intervention through Facebook to strengthen Self-esteem in nursing students. Rev Latino-Am Enfermagem. 2020;28:e3237. DOI: http://dx.doi. org/10.1590/1518-8345.3215.3237
23. Sousa CS, Turrini RNT. Development of an educational mobile application for patients submitted to orthognathic surgery. Rev Latino-Am Enfermagem. 2019;27:e3143. DOI: http://dx.doi. org/10.1590/1518-8345.2904.3143

24. Castro FSF, Dias DMV, Higarashi IH, Scochi CGS, Fonseca LMM. Avaliação da interação estudantetecnologia educacional digital em enfermagem neonatal. Rev Esc Enferm USP. 2015;49(1):114-21. DOI: 10.1590/S0080-623420150000100015

25. Cappellozza A, Moraes GHSM. Os países diferem entre si no acesso a internet? Rev eletrôn negócios internacionais [Internet]. 2014 [cited 2020 Jun 13];9(1):61-80. Available from: https://www. redalyc.org/articulo.oa?id $=557557882005$

Recebido: 4 de junho de 2020

Aprovado: 4 de julho de 2020

Publicado: 24 de novembro de 2020

A Revista Baiana de Enfermagem utiliza a Licença Creative Commons - Atribuição-NãoComercial 4.0 Internacional.

https://creativecommons.org/licenses/by-nc/4.0/

Este artigo é de acesso aberto distribuído sob os termos da Licença Creative Commons (CC BY-NC).

Esta licença permite que outros remixem, adaptem e criem a partir do seu trabalho para fins não comerciais. Embora os novos trabalhos tenham de lhe atribuir o devido crédito e não possam ser usados para fins comerciais, os usuários não têm de licenciar esses trabalhos derivados sob os mesmos termos. 